\title{
Simulation of Hardness Ratio grids for the ROSAT PSPC
}

\author{
Michael J. Carr \\ Dublin Institue of Technology, Bolton St. Dublin 1 \\ and Dunsink Observatory. \\ Castleknock, Dublin 15. \\ Evert J.A. Meurs \\ Dunsink Observatory, Castleknock, Dublin 15 and \\ John Cunniffe \\ National University of Ireland,University road, Galway
}

michael.carr@dit.ie

\begin{abstract}
Spectra are modeled for observations with the ROSAT PSPC, using 3 models namely Thermal Bremsstrahlung, Raymond-Smith and an Absorbed Power law. For each model a range of parameters are modeled. Hardness Ratios are calculated for each simulation and a grid of HR1 versus HR2 is produced for each of the three models. Hardness Ratios, from observations which do not have enough spectral counts to fit spectra directly, may be compared with the grid to set limits on observational parameters e.g temperature.
\end{abstract}

Keywords: x-rays, data-analysis,

\section{INTRODUCTION}

When observations are made in the X-ray it is possible to produce spectra of these objects. Xray spectra of these observations may be modeled and fitted to observations for objects with enough spectral counts ( $\geq 1000$ photons). From these spectra we can derive information about astronomical objects i.e., temperature, amount of neutral hydrogen present, abundances etc. We can also use Hardness Ratios (the X-ray equivalent of optical colours) that compare the photon counts in selected bands of the spectra, to derive information about objects. These are especially important for observations with low counts $(\leq 1000)$ photons. ROSAT Hardness Ratio 1 (HR1) and Hardness Ratio 2 (HR2) are defined as Zimmermann et al. 1992

$$
\begin{aligned}
& H R 1=\frac{(52-201)-(11-41)}{(11-41)+(52-201)} \\
& H R 2=\frac{(91-201)-(52-90)}{(52-90)+(91-201)}
\end{aligned}
$$

where the channel numbers 11-41 correspond to the number of photons contained in the energy interval $0.11-0.41 \mathrm{keV}$. These give us ratios of the soft and hard part of the spectrum. The channels 42-51 are omitted as ROSAT is not very sensitive in this energy range. HRs are especially useful for maximising the extraction of information from the ROSAT data archive and for work on less luminous objects. In this work we simulate spectra for three different spectral models, namely the Absorbed Power law, Raymond-Smith and Thermal Bremsstrahlung for a range of parameters. Hardness ratios are then calculated for these spectra producing a grid of HR1 versus HR2. Using this grid it is then possible to relate observed values of HR1 and HR2 to actual physical parameters. 


\section{SPECTRAL SIMULATIONS}

The modeling was done using the EXSAS Zimmermann et al.1992 software analysis system. For the purpose of our simulations we use 100,000 counts. All these simulations use the default detector response matrix and the effective area table for the PSPCb camera on ROSAT.

\section{ABSORBED POWER LAW MODEL}

Spectra are simulated for Absorbed Power law models with a range of values of absorbing $N_{H}$ (0.05 - $1.5 \times 10^{20} \mathrm{~cm}^{-2}$, in steps of $0.05 \times 10^{20} \mathrm{~cm}^{-2}$ ) and the Photon Index $\Gamma$ (from -0.25 through to -4 in steps of 0.25$)$. Hardness Ratios are calculated for these spectra. Figure 1 shows a HR1 versus HR2 plot for the absorbed power law model with values of HR1 ranging from -1 to 1 and $\mathrm{HR} 2$ ranging from -0.5 to 0.8 .

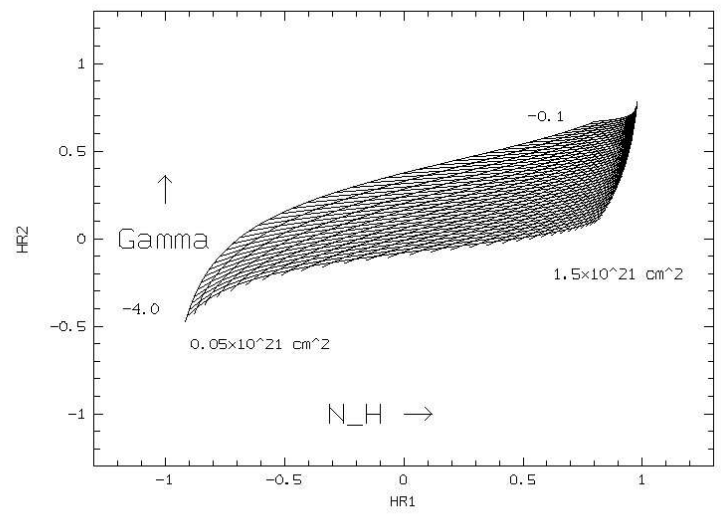

FIGURE 1: Simulated grid for Absorbed power law model. $N_{H}$ increases from $0.05-1.5 \times 10^{20} \mathrm{~cm}^{-2}$, in steps of $0.05 \times 10^{20} \mathrm{~cm}^{-2}$ on going from left to right. The Photon Index $\Gamma$ increases from -0.25 to -4 in steps of 0.25 om going from bottom to top.

\section{RAYMOND-SMITH MODEL}

Spectra are simulated for Raymond-Smith models with a range of values of absorbing $N_{H}$ $\left(0.05-1.5 \times 10^{20} \mathrm{~cm}^{-2}\right.$, in steps of $\left.0.05 \times 10^{20} \mathrm{~cm}^{-2}\right), \mathrm{T}(0.1-3 \mathrm{keV}$ in steps of $0.1 \mathrm{keV}$ and $3-16$ $\mathrm{keV}$ in steps of $0.5 \mathrm{keV}$ ) and redshift (0.0 - 0.8 in steps of 0.2$)$. Hardness Ratios are calculated for these spectra. Figures 2 and 3 show HR1 versus HR2 plot for the Raymond-Smith model for $\mathrm{z}=0$ and $z=0.8$ respectively. Values of HR1 range from -1 to 1 and values of HR2 range from -1 to 0.6 .

\section{THERMAL BREMSSTRAHLUNG}

Spectra are simulated for Thermal Bremsstrahlung models using the same parameters as for the Raymond-Smith models above. Hardness Ratios are calculated for these spectra. Figures 4 and 5 show HR1 versus HR2 plot for the Thermal Bremsstrahlung model for $z=0$ and $z=0.8$ respectively. Values of HR1 range from -1 to 1 and values of HR2 range from -1 to 0.6 .

\section{CALIBRATION}

To test the reliability of our grids we use them to derive spectral values from observations with good counts ( $>1000$ photons) and good spectral fits. We chose one observation corresponding to the Power Law fit and the Raymond-Smith fit. We are currently in the process of doing this for the Thermal Bremsstrahlung model. 


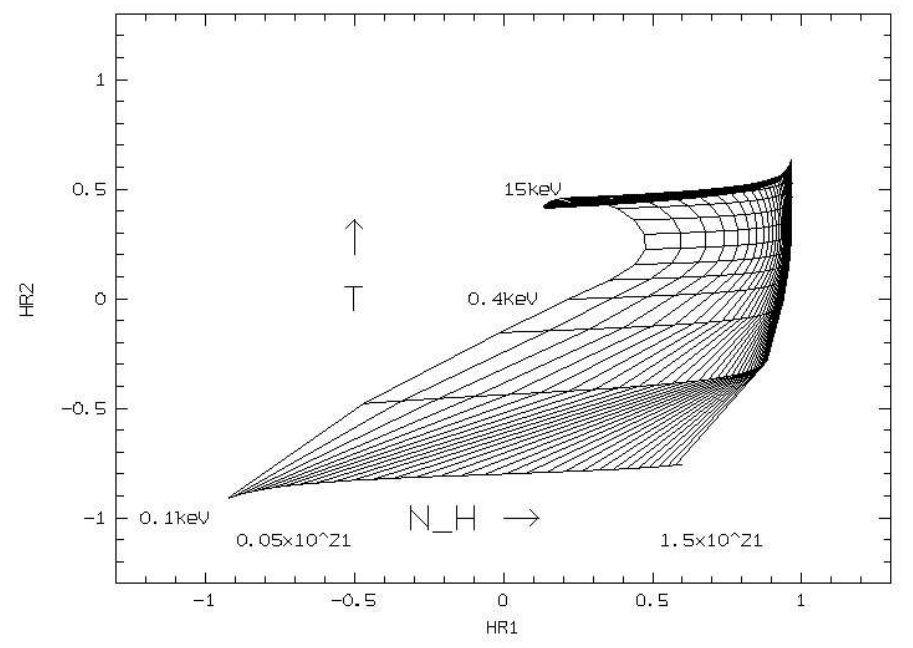

FIGURE 2: Simulated grid for RS model with $\mathrm{z}=0 . N_{H}$ increases from $0.05-1.5 \times 10^{20} \mathrm{~cm}^{-2}$, in steps of $0.05 \times 10^{20} \mathrm{~cm}^{-2}$ on going from left to right. The temperature increases from $0.1-3 \mathrm{keV}$ in steps of $0.1 \mathrm{keV}$ and from $3-16 \mathrm{keV}$ in steps of 0.5 on going from bottom to top.

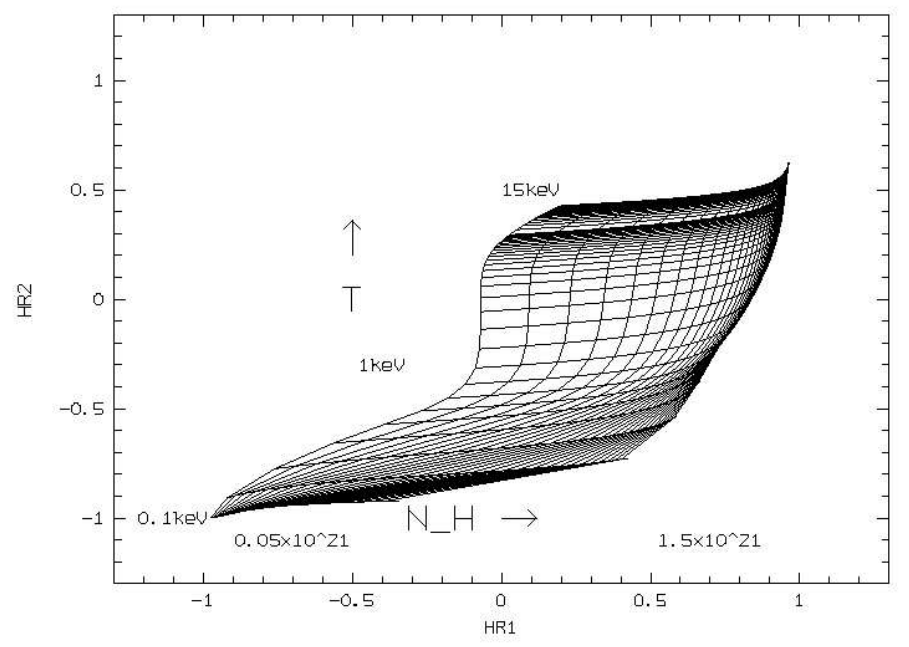

FIGURE 3: Simulated grid for RS model with $\mathrm{z}=0.8 . N_{H}$ increases from $0.05-1.5 \times 10^{20} \mathrm{~cm}^{-2}$, in steps of $0.05 \times 10^{20} \mathrm{~cm}^{-2}$ on going from left to right. $N_{H}$ increases from $0.05-1.5 \times 10^{20} \mathrm{~cm}^{-2}$, in steps of $0.05 \times 10^{20} \mathrm{~cm}^{-2}$ on going from left to right. The temperature increases from $0.1-3 \mathrm{keV}$ in steps of 0.1 $\mathrm{keV}$ and from $3-16 \mathrm{keV}$ in steps of 0.5 on going from bottom to top.

\subsection{Data Reduction}

The ROSAT PSPC data were analyzed using the EXSAS/MIDAS data reduction package (Zimmermann 1992), with corrections for bad times and vignetting. Images are created in the 0.11-2.35 keV energy range ,binned in 15 " pixels. A source detection is carried out on the PSPC images which produces a list of sources with a maximum likelihood of $\geq 10$. Their coordinates are compared with the target list and only those detections with an offset less than 2 " are accepted. Hardness Ratios are then extracted from these observations and used to derive spectral values using our simulated grids. 


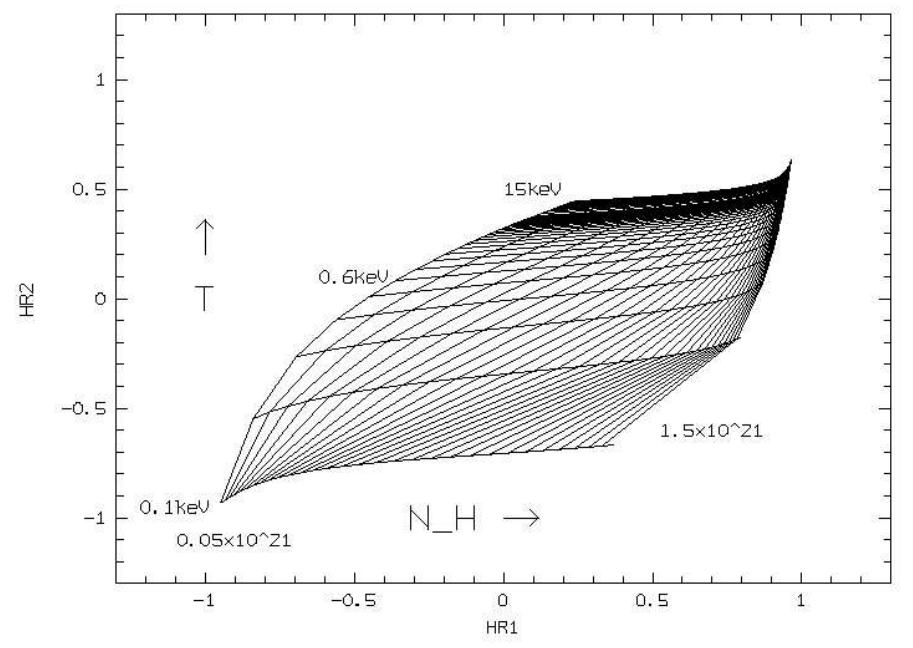

FIGURE 4: Simulated grid for the Thermal Bremsstrahlung model with $\mathrm{z}=0 . N_{H}$ increases from $0.05-1.5 \times$ $10^{20} \mathrm{~cm}^{-2}$, in steps of $0.05 \times 10^{20} \mathrm{~cm}^{-2}$ on going from left to right. $N_{H}$ increases from $0.05-1.5 \times 10^{20} \mathrm{~cm}^{-2}$, in steps of $0.05 \times 10^{20} \mathrm{~cm}^{-2}$ on going from left to right. The temperature increases from $0.1-3 \mathrm{keV}$ in steps of $0.1 \mathrm{keV}$ and from $3-16 \mathrm{keV}$ in steps of 0.5 on going from bottom to top.

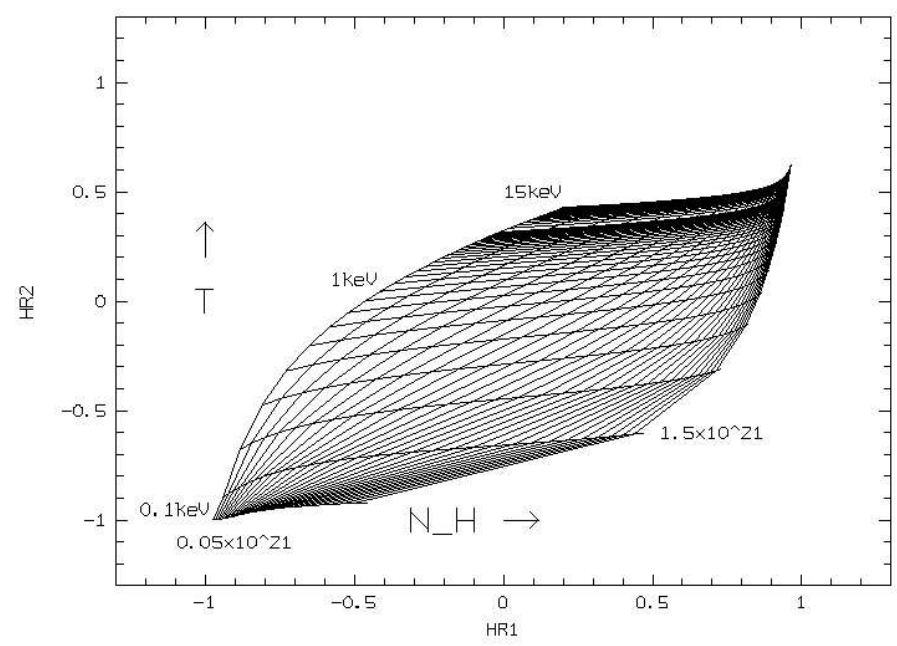

FIGURE 5: Simulated grid for the Thermal Bremsstrahlung model with $\mathrm{z}=0.8 . N_{H}$ increases from $0.05-1.5 \times$ $10^{20} \mathrm{~cm}^{-2}$, in steps of $0.05 \times 10^{20} \mathrm{~cm}^{-2}$ on going from left to right. $N_{H}$ increases from $0.05-1.5 \times 10^{20} \mathrm{~cm}^{-2}$, in steps of $0.05 \times 10^{20} \mathrm{~cm}^{-2}$ on going from left to right. The temperature increases from $0.1-3 \mathrm{keV}$ in steps of $0.1 \mathrm{keV}$ and from $3-16 \mathrm{keV}$ in steps of 0.5 on going from bottom to top.

\subsection{Power law Calibration}

The ROSAT PSPC observation of the QSO RX J1333.7+3803 is used to calibrate our power law grid. Dewangan et al.(2002) analyzed this data and found that it is well fitted (reduced $\chi^{2}=0.88$ ) by a power law with spectral index $\Gamma=2.28_{-0.27}^{+0.31}$ and with an absorbing hydrogen column density of $0.37_{-0.37}^{+0.5} \times 10^{20} \mathrm{~cm}^{-2}$. Extracting hardness ratios from the PSPC observation we obtain HR1 $=-0.52 \pm 0.04$ and HR2 $=0.04 \pm 0.015$. Inputing these values into our power law gird we get a value of $-2.86_{-0.3}^{+0.1}$ for the spectral index $\Gamma$ with an absorbing hydrogen column density of 
$1.33_{-0.5}^{+0.8} \times 10^{20} \mathrm{~cm}^{-2}$. Thus the values returned by the grid method are consistent with the results of a detailed spectral analysis.

\subsection{Raymond-Smith Calibration}

Donahue \& Stocke 1995 observed the Cluster MS0735.6+7421 with the ROSAT PSPC. They measured a temperature of $2.4_{-0.8}^{+2.2} \mathrm{keV}$ with an absorbing hydrogen column density of $3.4_{-0.5}^{+0.5} \times$ $10^{20} \mathrm{~cm}^{-2}$. Extracting hardness ratios from the observation we obtain $\mathrm{HR} 1=0.61 \pm 0.03$ and $\mathrm{HR} 2=0.26 \pm 0.02$. Inputting these values into our Raymond-Smith grid we obtain a temperature of $1.2_{-0.04}^{+0.40}$ with an absorbing column density of $1.9_{-0.01}^{+2.1} \times 10^{20} \mathrm{~cm}^{-2}$ consistent with the measurements of Donahue \& Stocke

\subsection{Previous HR grids}

Kahabka [2002] simulates hardness ratio grids as a means of distinguishing AGNs from XRBs using hardness ratios. This grid uses 3 values of $\Gamma$ and 5 values of absorbing $N_{H}$. The results of Kahabka are found to be consistent with our Grid.

\section{CONCLUSIONS}

We have modelled HR grids for three models, namely absorbed power law, Raymond-Smith and thermal bremsstrahlung. These grids have been calibrated against X-ray observations for both the power law and Raymond-Smith models and we are currently in the process of calibrating these models for the thermal bremsstrahlung model. The values returned by our grid are found to be consistent with the results of a detailed spectral analysis. We have produced a tool which allows us to set limits on observational parameters e.g temperature using observations which do not have enough spectral counts to fit spectra directly.

\section{REFERENCES}

[1] Dewangan G.C., et al. MNRAS,337,693

[2] Dickey J.M., \& Lockman F.J. ,1990 ARA \&A,28,215

[3] Donahue, M \& Stocke J.T.,1995 Ap.J,468,79.

[4] Kahabka, P;2002 A\&A, 388, 100-112

[5] Zimmermann, H. U.; Belloni, T.; Boese, G.; Izzo, C.; Kahabka, P.; Schwentker, O. 1992 In Proc. European ISY meeting. Symposium Space Sciences. 